Pacific

Journal of

Mathematics

LOCAL VERSUS GLOBAL PEAK SETS IN REAL-ANALYTIC CONVEX BOUNDARIES

RACHID BELHACHEMI 


\title{
LOCAL VERSUS GLOBAL PEAK SETS IN REAL-ANALYTIC CONVEX BOUNDARIES
}

\author{
RACHID BELHACHEMI
}

Suppose $D \subset \subset \mathbb{C}^{3}$ is a convex domain with real-analytic boundary. Then a compact subset $K$ of $\partial D$ is locally a peak set for $A^{\infty}(D)$ if and only if $K$ is a peak set for $A^{\infty}(D)$.

\section{Introduction.}

\subsection{Notations and Definitions.}

Throughout most of this paper, $D$ will be a smoothly bounded domain in $\mathbb{C}^{n}$. We denote by $A^{\infty}(D)$ the set of holomorphic functions in $D$ which have $C^{\infty}$-extension to $\bar{D}$.

A closed subset $K \subset \partial D$ is a peak set for $A^{\infty}(D)$ if there exists a function $f \in A^{\infty}(D)$ so that $f=1$ on $K$ and $|f|<1$ on $\bar{D} \backslash K$. $K$ is locally a peak set for $A^{\infty}(D)$ if for each $p \in K$, there exists a neighborhood $V$ of $p$ so that $K \cap \bar{V}$ is a peak set for $A^{\infty}(D)$.

It is easy to see that a closed subset $K \subset \partial D$ is a peak subset for $A^{\infty}(D)$ if and only if there exists a function $g \in A^{\infty}(D)$ such that $g=0$ on $K$ and $\operatorname{Reg}>0$ on $\bar{D} \backslash K$. Such a function $g$ is called a strong support function for $K$.

We denote by $T_{p}(M)$ the real tangent space to a smooth manifold $M$ at the point $p \in M$. For a point $p \in M$, the complex tangent space of $M$ at $p$ is the vector space

$$
T_{p}^{\mathbb{C}}(M)=T_{p}(M) \cap J\left\{T_{p}(M)\right\} .
$$

Here $J$ is the almost complex structure corresponding to multiplication by $i$. $T_{p}^{\mathbb{C}}(M)$ is the maximal complex subspace of $T_{p}(M)$, of complex dimension $n-1$ if $M=\partial D$.

A smooth submanifold $M \subseteq \partial D$ is integral at $p \in M$ if $T_{p}(M) \subseteq$ $T_{p}^{\mathbb{C}}(\partial D) . M$ is an integral manifold if it is integral at each point $p \in M$. We say $M$ is totally real if $T_{p}^{\mathbb{C}}(M)=\{0\}$ for every $p \in M$.

A domain $D \subset \mathbb{C}^{n}$ has $C^{\infty}$ boundary at $p \in \partial D$ if there is a $C^{\infty}$ function $r: U \rightarrow \mathbf{R}$ defined on a neighborhood $U$ of $p$ so that $\nabla r(p) \neq 0$ and $D \cap U=\{z \in U: r(z)<0\}$. Such a function $r$ is called a defining function for $D$ at $p$. From here on, $r$ denotes a defining function for $D$. 
We say $D$ is (Levi) pseudoconvex at $p$ if

$$
L_{r}(p, w)=\sum_{j, k=1}^{n} \frac{\partial^{2} r}{\partial z_{j} \partial \bar{z}_{k}}(p) w_{j} \bar{w}_{k} \geq 0
$$

for all $w \in T_{p}^{\mathbb{C}}(\partial D) . L_{r}(p, w)$ is called the Levi form or the complex Hessian of $r$.

Let $D$ be pseudoconvex at $p$. The point $p$ is said to be strongly pseudoconvex if the Levi form is positive whenever $w \neq 0, w \in T_{p}^{\mathbb{C}}(\partial D)$.

The point $p$ is said to be weakly pseudoconvex if the Levi form is zero for some $w \neq 0, w \in T_{p}^{\mathbb{C}}(\partial D)$.

We denote by $w(\partial D)$ the set of weakly pseudoconvex boundary points.

A domain is called pseudoconvex (resp. strongly pseudoconvex) if all its boundary points are pseudoconvex (rep. strongly pseudoconvex).

For $p \in \partial D$, we let $N_{p}$ denote the null space in $T_{p}^{\mathbb{C}}(\partial D)$ of the Levi form at $p$. This, as well as notions defined above are independent of the defining function. $D^{\alpha}$ will denote the differential operator $\frac{\partial^{|\alpha|}}{\partial x_{1}^{\alpha_{1}} \partial x_{2}^{\alpha_{2}} \cdots \partial x_{N}^{\alpha_{N}}}$ in $\mathbf{R}^{N}$, where $\alpha=\left(\alpha_{1}, \ldots, \alpha_{N}\right)$ and $|\alpha|=\alpha_{1}+\cdots+\alpha_{N}$.

We will denote by $d(z, M)$ the Euclidean distance from $z$ to a manifold $M$. Int $(A)_{B}$ will denote the interior of $A$ in $B$.

\subsection{Main result.}

Our main result is:

Theorem 16. Suppose $D \subset \subset \mathbb{C}^{3}$ is a convex domain with real-analytic boundary. Then a compact subset $K$ of $\partial D$ is locally a peak set for $A^{\infty}(D)$ if and only if $K$ is a peak set for $A^{\infty}(D)$.

\subsection{Historical background.}

The subject of peak sets has been studied in recent years by several authors [4], [5], [9], [10], [12], [14], [18]. If $D$ is the unit disc in the complex plane, B.A. Taylor and D.L. Williams $[\mathbf{1 8}]$ proved that the only peak sets for $A^{\infty}(D)$ are the finite subsets of $\partial D$.

In $\mathbb{C}^{n}, n \geq 2$, the situation is different. In the strongly pseudoconvex case Hakim and Sibony [10], Chaumat and Chollet [4], [5], and Fornaess and Henriksen [9] gave the following characterization of locally peak sets for $A^{\infty}(D)$.

Theorem 1. Let $D$ be a bounded strongly pseudoconvex domain in $\mathbb{C}^{n}$ with smooth boundary and $K$ a closed subset of $\partial D$. The following conditions are equivalent:

(1) $K$ is locally a peak set for $A^{\infty}(D)$.

(2) $K$ is locally contained in an $(n-1)$-dimensional totally real submanifold of $\partial D$, which is integral at each point of $K$. 
(3) $K$ is locally contained in an $(n-1)$-dimensional totally real integral submanifold of $\partial D$.

(4) $K$ is a peak set for $A^{\infty}(D)$.

For weakly pseudoconvex domains in $\mathbb{C}^{n}$, the aforementioned characterization of peak sets does not hold in general, including those which are convex with real-analytic boundary in $\mathbb{C}^{2}$. In [14], Noell showed that there exists a convex domain $D$ with real-analytic boundary in $\mathbb{C}^{2}$ and a peak set $K$ for $A^{\infty}(D)$ which is not contained in any smooth curve. He also showed that there exists a convex domain $D$ with real-analytic boundary in $\mathbb{C}^{2}$ and an integral curve $M \subset \partial D$ so that $M \cap \bar{U}$ is not a peak set for $A^{\infty}(D)$ for any neighborhood $U$ of $0 \in M$. Hence the implications $(1) \Rightarrow(2)$ and $(3) \Rightarrow(1)$ do not hold for weakly pseudoconvex domains. The implication $(1) \Rightarrow(4)$ breaks down in general. In fact, Fornaess in [7] constructed a bounded pseudoconvex domain $D \subset \mathbb{C}^{2}$ with real-analytic boundary, and in [14], Noell proved that $K=w(\partial D)$ is locally a peak set for $A^{\infty}(D)$, but $K$ is not a peak set for $A^{\infty}(D)$. There is however, a positive result for the implication $(1) \Rightarrow(4)$ in convex domains with real-analytic boundaries in $\mathbb{C}^{2}$. This result is due to Noell, and appears in [14]. Our goal in this paper is to extend the implication $(1) \Rightarrow(4)$ to convex domains with real-analytic boundaries in $\mathbb{C}^{3}$.

At this point, one asks the following question: What makes locally peak sets globally peak sets in convex domains with real-analytic boundaries in $\mathbf{C}^{2}$ ? The answer to this question depends on two criteria.

First, Noell in [14] imposed the (NP) condition on the domain which guarantees that we need only to patch peak functions at strongly boundary points, and this is always achieved for such points by Theorem $1((1) \Rightarrow(4))$. He also showed that every convex domain with real-analytic boundary in $\mathbf{C}^{2}$ satisfies the (NP) property. More precisely, Noell defined this "nonpropagation" property as follows: Suppose $D \subset \subset \mathbb{C}^{2}$ is a pseudoconvex domain with real-analytic boundary. We say that $D$ has property (NP) if there does not exist a real-analytic integral curve contained in $w(\partial D)$.

Second, a decomposition of $w(\partial D)$ in $\mathbb{C}^{2}$ given by Fornaess and Overlid in $[8]$ is rather simple, and using this in conjunction with the (NP) property, we only need to patch peak functions away from isolated sets. For more details, we refer the reader to [14].

However, as the example below shows, bounded convex domains with real-analytic boundaries in $\mathbb{C}^{3}$ need not have the (NP) property.

Example 2. Let $D=\left\{\left(z_{1}, z_{2}, z_{3}\right) \in \mathbb{C}^{3}:\left|z_{1}\right|^{2}+\left|z_{2}\right|^{2}+\left|z_{3}\right|^{4}<1\right\}$. It is clear that $D$ is convex with real-analytic boundary, however

$$
w(\partial D)=\left\{\left(z_{1}, z_{2}, z_{3}\right) \in \partial D: z_{3}=0 \text { and }\left|z_{1}\right|^{2}+\left|z_{2}\right|^{2}=1\right\}
$$


contains a real-analytic integral curve, and therefore $D$ does not have the (NP) condition.

\section{Linear regularity.}

Our first task in this paper is to find a local decomposition of $w(\partial D)$ which holds for convex domains in $\mathbb{C}^{n}$ with real-analytic boundaries. The main ingredient in the proof of Theorem 5 is to use the condition of "Linear regularity" (Definition 3) which is the natural generalization of the "NP" property introduced by Noell in [14]. Moreover, it is shown in [15] that every bounded convex domain in $\mathbb{C}^{n}$ with real-analytic boundary is linearly regular. For completeness, a proof of this fact is included in this section (Theorem 4).

Definition 3. Suppose $D \subset \subset \mathbb{C}^{n}$ is a pseudoconvex domain with smooth boundary. We say $D$ is "linearly regular" if there does not exist a smooth curve $\gamma$ in $\partial D$ so that $\gamma^{\prime}(t) \in N_{\gamma(t)}$ for all $t \in I$, where $I \subset \mathbf{R}$ is an interval.

Theorem 4 ([15]). Suppose $D \subset \subset \mathbb{C}^{n}$ is a convex domain with real-analytic boundary. Then $D$ is linearly regular.

Proof. ${ }^{1}$ Assume to the contrary that there exists a smooth curve $\gamma$ in $\partial D$ so that $\gamma^{\prime}(t) \in N(\gamma(t))$ for all $t$. From this, we will show that $\partial D$ contains a line segment, and this will be a contradiction.

Assume $\gamma$ is defined on an interval $I \subset \mathbf{R}$. Let $n(t)=\nabla r(\gamma(t))$, and $H(t)$ denote the $(2 n \times 2 n)$ matrix of real second-order partial derivatives of $r$ evaluated at $\gamma(t)$. Assume $\|n\|=1$.

Let $\langle$,$\rangle denote the real inner product on \mathbb{C}^{n}$ by identifying vectors in $\mathbb{C}^{n}$ with vectors in $\mathbf{R}^{2 n}$.

Claim. $n(t)$ is constant for all $t \in I$.

Fix $t \in I$. Choose complex coordinates $z_{j}=x_{j}+i y_{j}, 1 \leq j \leq n$ so that $\gamma^{\prime}(t)=\left.\frac{\partial}{\partial x_{1}}\right|_{\gamma(t)}$ and $n(t)=\left.\frac{\partial}{\partial x_{n}}\right|_{\gamma(t)}$. The Chain Rule gives

$$
n^{\prime}(s)=H(s) \gamma^{\prime}(s)
$$

for all $s \in I$.

Using the convexity of $D$, and since $\gamma^{\prime}(t) \in N_{\gamma(t)}$, we get $\frac{\partial^{2} r}{\partial x_{1}^{2}}(\gamma(t))=0$. Also the convexity of $D$ gives $\frac{\partial^{2} r}{\partial x_{1} \partial x_{j}}(\gamma(t))=\frac{\partial^{2} r}{\partial x_{1} \partial y_{k}}(\gamma(t))=0$, when $1 \leq j \leq n-1,1 \leq k \leq n$. So,

$$
\left\langle H(t) \gamma^{\prime}(t), \mu\right\rangle=0
$$

\footnotetext{
${ }^{1}$ I wish to thank Professor Alan Noell for his useful advice, and for providing a lot of inspiration for much of my work.
} 
when $\mu \in T_{\gamma(t)}(\partial D)$. Using this and (1), we conclude that $n^{\prime}(t)$ is perpendicular to $T_{\gamma(t)}(\partial D)$.

Now, since $\langle n(s), n(s)\rangle \equiv 1$ for all $s \in I$, differentiation of both sides yields $\left\langle n^{\prime}(s), n(s)\right\rangle \equiv 0$, which implies that $n^{\prime}(s)$ is orthogonal to $n(s)$ for all $s \in I$. Because $n^{\prime}(t)$ is orthogonal to $T_{\gamma(t)}(\partial D)$, we have $n^{\prime}(t) \equiv 0$, and hence the claim follows.

Let $n(t)=\lambda$ for all $t \in I$, where $\lambda$ is a constant. Define the function $g$ as follows:

For $s, t \in I$, put

$$
g(s, t)=\langle\gamma(s)-\gamma(t), \lambda\rangle .
$$

Then $\frac{\partial g}{\partial s}=\frac{\partial g}{\partial t} \equiv 0$, so $g$ is a constant, since $g(s, s)=0$ we get $g \equiv 0$. Thus $(\gamma(s)-\gamma(t)) \in T_{\gamma(t)}(\partial D)$, when $s, t \in I$. However, since $D$ is convex, then the line segment through $\gamma(s)$ and $\gamma(t)$ must lie in $\partial D$. But this is a contradiction, since $D$ is bounded with real-analytic boundary. Thus $D$ is linearly regular.

\section{Preliminary Theorem.}

Our major goal in this section is to obtain a stratification of $w(\partial D)$ in $C^{n}$ for convex domains with real-analytic boundaries (Theorem 5 ). In fact, the theorem holds for real-analytic domains which are linearly regular. Theorem 5 shows that the analysis on convex domains with real-analytic boundaries in $\mathbb{C}^{n}$ is somewhat similar to strongly pseudoconvex domains.

Theorem 5. Suppose $D \subset \subset \mathbb{C}^{n}$ is a convex domain with real-analytic boundary. Then for each $p \in w(\partial D)$, there exists a neighborhood $U$ of p so that:

(a) $w(\partial D) \cap U=\bigcup_{j=0}^{2 n-3} S_{j}$, where each $S_{j}$ is a finite disjoint union of $j$-dimensional real-analytic $\mathrm{CR}$ submanifolds of $\partial D \cap U$. Furthermore, for all $q \in S_{j}, T_{q}\left(S_{j}\right) \cap N_{q}=\{0\}$.

(b) If $S$ is a component of some $S_{j}$ and $T_{q}(S) \subset T_{q}^{\mathbb{C}}(\partial D)$ for some $q \in S$, then $S$ is an integral submanifold of $\partial D \cap U$.

(c) $S_{j}$ is closed in $\partial D \backslash\left(\bigcup_{i=0}^{j-1} S_{i}\right), j=1, \ldots, 2 n-3$.

Before we embark on the proof of Theorem 5 , we introduce the necessary definitions and facts needed in this section.

Suppose $S$ is a smooth submanifold of $\mathbb{C}^{n}$. We say $S$ is a CR manifold if $\operatorname{dim}_{\mathbb{C}} T_{p}^{\mathbb{C}}(S)$ is constant on $S$ as a function of $p$. If $S \subset \partial D$ is a CR manifold, we say $S$ has holomorphic dimension zero if for all $p \in S$ and all nonzero $\left(t_{1}, \ldots, t_{n}\right) \in T_{p}^{\mathbb{C}}(S)$ we have

$$
\sum_{j, k=1}^{n} \frac{\partial^{2} r}{\partial z_{j} \partial \bar{z}_{k}}(p) t_{j} \bar{t}_{k}>0 .
$$


Let $S$ be a real-analytic manifold. We say $S^{\prime} \subset S$ is a real-analytic subset of $S$ if for every $p \in S$ there exists a neighborhood $U$ of $p$ and a real-analytic map

$$
F: U \rightarrow \mathbf{R}^{m}
$$

so that

$$
S^{\prime}=\{q \in U: F(q)=0\} .
$$

Now, we state Lojaciewicz's Theorem [13] which tells us that a realanalytic variety can be stratified into submanifolds of lower dimensions.

Theorem 6. Suppose that $f$ is a non-constant real-analytic function defined in a neighborhood $U \subset \mathbf{R}^{n}$ of the origin. Assume that the zero set $Z$ of $f$ in $U$ is nonempty. Then $Z$ has the following decomposition:

$$
Z=S_{n-1} \cup S_{n-2} \cup \cdots \cdots \cup S_{0}
$$

where each $S_{j}(1 \leq j \leq n-1)$ is a finite disjoint union of $j$-dimensional real-analytic submanifolds. Furthermore, $S_{j}$ is closed in $Z \backslash\left(\cup_{i=0}^{j-1} S_{i}\right), j=$ $1, \ldots, n-1$.

Proof of Thoerem 5. Using a result of Diederich and Fornaess [6], we can find real-analytic submanifolds $F_{1}, \ldots, F_{\tau}$ in $\partial D$ of holomorphic dimension zero so that

$$
w(\partial D)=\bigcup_{m=1}^{\tau} F_{m},
$$

with each $F_{k}$ closed in $\partial D \backslash\left(\bigcup_{i=1}^{\tau} F_{i}\right), k=1, \ldots, \tau$. The result in [6] is true for pseudoconvex domains in $\mathbb{C}^{n}$ with real-analytic boundary.

Let $V_{k}=\left\{z \in \partial D: \operatorname{dim}_{\mathbb{C}} N_{z} \leq k\right\}, k=1, \ldots, n-1$. Then each $V_{k}$ is a closed real-analytic subvariety of $\partial D$.

Put $W_{k}=V_{k} \backslash V_{k-1}$, and note that $w(\partial D)=\bigcup_{k=1}^{n-1} W_{k}$.

Fix $m$, and drop the subscript from $F_{m}$.

First, we note that $F$ could not be of dimension $2 n-1$, since $F$ is of holomorphic dimension zero.

Lemma 7. Let $F$ be as above. Then $\operatorname{dim}_{\mathbf{R}} F \leq 2 n-3$.

Proof. Assume for a contradiction that $\operatorname{dim}_{\mathbf{R}} F=2 n-2$.

We note that since $F=\left(W_{1} \cup W_{2} \cup \cdots \cup W_{n-1}\right) \cap F$, then $\operatorname{Int}\left(W_{i} \cap F\right)_{F} \neq$ $\emptyset$ for some $i(1 \leq i \leq n)$. We claim that $\operatorname{Int}\left(W_{i} \cap F\right)_{F}=\emptyset$ for all $i>1$.

Suppose not, and let $q^{\prime} \in \operatorname{Int}\left(W_{i} \cap F\right)_{F}$ for some $i>1$. Then there exists a neighborhood $U^{\prime}$ of $q^{\prime}$ in $F$ so that $U^{\prime} \subset W_{i} \cap F$. Since $T_{q^{\prime}}^{\mathbb{C}}\left(U^{\prime}\right) \cap N_{q^{\prime}}=\{0\}$, and $\operatorname{dim}_{\mathbb{C}} T_{q^{\prime}}^{\mathbb{C}}\left(U^{\prime}\right) \geq n-2$, this implies that $\operatorname{dim}_{\mathbb{C}} T_{q^{\prime}}^{\mathbb{C}}(\partial D) \geq n-2+2=n$. This is impossible, since $\operatorname{dim}_{\mathbb{C}} T_{q^{\prime}}^{\mathbb{C}}\left(U^{\prime}\right)=n-1$. Thus the claim is true, and we conclude that int $\left(W_{1} \cap F\right)_{F} \neq \emptyset$. 
Now, choose a nonzero real-analytic vector field $X$ so that if $q \in W_{1}$ then $X(q)$ spans $N_{q}$ over $\mathbb{C}$. For $q \in \operatorname{Int}\left(W_{1} \cap F\right)_{F}$, put

$$
L_{q}=T_{q}(F) \cap N_{q} .
$$

Observe that $L_{q}$ is a real-subspace of $T_{q}(F)$, and $L_{q}$ is of real dimension at most 1 , because $F$ is of holomorphic dimension zero.

Suppose $\operatorname{dim}_{\mathbf{R}} L_{q_{0}}=0$ for some $q_{0} \in \operatorname{Int}\left(F \cap W_{1}\right)_{F}$. Then $T_{q_{0}}(F)$ contains no weakly pseudoconvex direction, but this is a contradiction, since $\operatorname{dim}_{\mathbf{R}} F=2 n-2$. Hence $\operatorname{dim}_{\mathbf{R}} L_{q}=1$ for all $q \in \operatorname{Int}\left(W_{1} \cap F\right)_{F}$.

Because a one-dimensional subbundle of the real tangent of a manifold is integrable, (integrable means closed under the Lie bracket operation) then by Frobenius theorem [3], there exists a smooth curve $\gamma \subset \partial D$ so that $T_{q}(\gamma)=L_{q}$ for all $q \in \operatorname{Int}\left(F \cap W_{1}\right)_{F}$, and hence $T_{q}(\gamma) \subset N_{q}$. Therefore $D$ is not linearly regular. But this is impossible, since by Theorem 4 , every convex domain with real-analytic boundary is linearly regular. Thus $\operatorname{dim}_{\mathbf{R}} F \leq$ $2 n-3$. This ends the proof of Lemma 7 .

Assume $\operatorname{dim}_{\mathbf{R}} F=2 n-3$.

Fix $k$, and let $p \in F \cap W_{k}$. For ease of notation, we drop the subscript from $W_{k}$. We will analyze the structure of $W \cap F$ in relation to the null space of the Levi form.

Choose nonzero real-analytic vector fields $\left\{X_{j}\right\}_{j=1}^{k}$ so that if $q \in W$ then $\left\{X_{j}(q)\right\}_{j=1}^{k}$ spans $N_{q}$.

Fix $j$, and let $M_{q}^{(j)}=\left\{\lambda X_{j}(q): \lambda \in \mathbb{C}\right\}$, then $M_{q}^{(j)}$ is a complex subspace of $N_{q}$ if $q \in W$. For $q \in F \cap W$, put

$$
L_{q}^{(j)}=T_{q}(F) \cap M_{q}^{(j)} .
$$

We observe that $L_{q}^{(j)}$ is a real subspace of $T_{q}(F)$, and $L_{q}^{(j)}$ is of real dimension at most 1 , since $F$ is of holomorphic dimension zero.

Let $S^{\prime}=\left\{q \in F \cap W: \operatorname{dim}_{\mathbf{R}} L_{q}^{(j)}=1\right\}$.

Lemma 8. Let $F$ and $S^{\prime}$ be as above. Then $\operatorname{Int}\left(S^{\prime}\right)_{F}=\emptyset$, and $S^{\prime}$ is a real-analytic subset of $F$.

Proof. Suppose that Int $\left(S^{\prime}\right)_{F} \neq \emptyset$, and let $q_{0} \in \operatorname{Int}\left(S^{\prime}\right)_{F}$. Choose a neighborhood $V$ of $q_{0}$ in $F$ so that $\operatorname{dim}_{R} L_{q}^{(j)}=1$ for all $q \in V$. By Frobenius Theorem [3], there exists a smooth curve $\gamma \subset \partial D$ so that $T_{q}(\gamma)=L_{q}^{(j)} \subset N_{q}$ for every $q \in \gamma \cap V$. This is impossible by linear regularity. So $\operatorname{Int}\left(S^{\prime}\right)_{F}=\emptyset$.

We will now show that $S^{\prime}$ is a real-analytic subset of $F$.

Let $\left\{v_{i}\right\}_{i=1}^{2 n-3}$ be nonzero real-analytic vector fields so that $\left\{v_{i}(q)\right\}_{i=1}^{2 n-3}$ spans $T_{q}(F)$ for all $q \in F$. Let $q \in S^{\prime}$, and consider the $(2 n-1) \times(2 n)$ 
matrix

$$
A_{q}=\left[\begin{array}{c}
v_{i}(q) \\
X_{j}(q) \\
J X_{j}(q)
\end{array}\right] \quad(1 \leq i \leq 2 n-3)
$$

It follows from elementary linear algebra that if $q \in F \cap W$, then

$$
\begin{aligned}
q \in S^{\prime} & \Longleftrightarrow \operatorname{Rank}\left(A_{q}\right) \leq 2 n-2 \\
& \Longleftrightarrow \operatorname{det}\left(B_{q}\right)=0 \\
& \quad \text { for every }(2 n-1) \times(2 n-1) \text { submatrix } B_{q} \text { of } A_{q} .
\end{aligned}
$$

Since $\operatorname{det}\left(B_{q}\right)$ is a real-analytic function, then $S^{\prime}$ is a real-analytic subset of $S$. This finishes the proof of Lemma 8.

Now, we will apply Theorem 6 to stratify the real-analytic set $S^{\prime}$ defined above.

For a small neighborhood $U$ of $p$, we may write

$$
S^{\prime} \cap U=\Gamma_{2 n-4} \cup \cdots \Gamma_{0},
$$

where each $\Gamma_{t},(0 \leq t \leq 2 n-4)$ is a finite disjoint union of $t$-dimensional real-analytic submanifolds of $\partial D$. One also notes that since $\Gamma_{t} \subset F$ and since $F$ is of holomorphic dimension zero, so is $\Gamma_{t}$ for all $t,(0 \leq t \leq 2 n-4)$.

Let $S^{\prime \prime}=(F \cap W) \backslash S^{\prime}$. Then $S^{\prime \prime}$ is an open subset of $F$, and $S^{\prime \prime}$ is of holomorphic dimension zero.

Since $\operatorname{dim}_{\mathbf{R}} L_{q}^{(j)}=0$ for all $q \in S^{\prime \prime}$, then $T_{q}\left(S^{\prime \prime}\right) \cap M_{q}^{(j)}=\{0\}$ for each $q \in S^{\prime \prime} \cap U$. Doing this for each $j=1, \ldots, k$, we get a manifold that we still denote $S^{\prime \prime}$ of dimension $2 n-3$ such that for each $q$ near $p$

$$
T_{q}\left(S^{\prime \prime}\right) \cap N_{q}=\{0\}
$$

Using (3), and since $\operatorname{dim}{ }_{R} S^{\prime \prime}=2 n-3$, it follows that for all $q$ near $p$

$$
\operatorname{dim} \mathbb{C}_{q} T_{q}^{\mathbb{C}}\left(S^{\prime \prime}\right)=n-2
$$

Hence $S^{\prime \prime}$ is a $C R$ submanifold of $\partial D \cap U$. Furthermore, $S^{\prime \prime}$ is not integral near $p$ by (3) and (4).

We obtain from (2) that $F \cap W \cap U=S^{\prime \prime} \cup \Gamma_{2 n-4} \cup \cdots \cup \Gamma_{0}$. Since this is true for all $m$ and $k$, we conclude that

$$
w(\partial D) \cap U=\Lambda_{2 n-3} \cup \Lambda_{2 n-4} \cup \cdots \Lambda_{0},
$$

where $\Lambda_{2 n-3}$ is a finite disjoint union of $(2 n-3)$-dimensional real-analytic $C R$ submanifolds of $\partial D \cap U$, with

$$
T_{q}\left(\Lambda_{2 n-3}\right) \cap N_{q}=\{0\}
$$

for all $q$ near $p$, and $\Lambda_{t}(0 \leq t \leq 2 n-4)$ are real-analytic submanifolds of $\partial D \cap U$ of holomorphic dimension zero. 
The next thing we will do is to stratify the $t$-dimensional submanifods of $\partial D \cap U,(0 \leq t \leq 2 n-4)$ so that their tangent spaces do not contain any weakly pseudoconvex directions.

Let $Z \subset w(\partial D)$ be a real-analytic submanifold of $\partial D \cap U$ of holomorphic dimension zero, and assume $\operatorname{dim}_{\mathbf{R}} Z=2 n-4$.

Put $Z^{\prime}=\left\{q \in Z: \operatorname{dim}_{\mathbf{R}} L_{q}^{\prime(j)}=1\right\}$ where, for $q \in Z \cap W$,

$$
L_{q}^{\prime}(j)=T_{q}(Z) \cap M_{q}^{(j)} .
$$

We argue as in Lemma 8 and deduce that $\operatorname{Int}\left(Z^{\prime}\right)_{Z}=\emptyset$, and $Z^{\prime}$ is a realanalytic subset of $Z$.

Let $Z^{\prime \prime}=\left\{q \in Z \cap W: T_{q}(Z) \subseteq T_{q}^{\mathbb{C}}(\partial D)\right\}$.

Lemma 9. Let $Z$ and $Z^{\prime \prime}$ be as above. Then $\operatorname{Int}\left(Z^{\prime \prime}\right)_{Z}=\emptyset$, and $Z^{\prime \prime}$ is a real-analytic subset of $Z$.

Proof. Suppose this is not the case, and let $q_{0} \in \operatorname{Int}\left(Z^{\prime \prime}\right)_{Z}$. Then there exists a neighborhood $U_{1}$ of $q_{0}$ in $Z$ so that $T_{q}\left(U_{1}\right) \subseteq T_{q}^{\mathbb{C}}(\partial D)$ for all $q \in U_{1}$. There are two cases to consider.

If $n>3$, then since $T_{q_{0}}\left(U_{1}\right) \subset T_{q_{0}}^{\mathbb{C}}(\partial D)$, it follows that $\operatorname{dim}_{\mathbb{C}} T_{q_{0}}^{\mathbb{C}}\left(U_{1}\right) \geq$ $n-3 \geq 1$, moreover since $U_{1}$ is an integral manifold, then we deduce from a result of Bedford and Fornaess [1] (Lemma 3, p. 287) that $T_{q_{0}}^{\mathbb{C}}\left(U_{1}\right) \subset N_{q_{0}}$. But this is impossible, since $U_{1}$ is of holomorphic dimension zero.

If $n=3$, then $\operatorname{dim}_{\mathbb{C}} T_{q}^{\mathbb{C}}\left(U_{1}\right) \leq 1$ for all $q \in U_{1}$.

Assume $U_{1}$ is totally real, and hence in a neighborhood of $q$. For $q_{1} \in$ $U_{1} \cap W$, define

$$
L_{q_{1}}^{\prime \prime}=T_{q_{1}}\left(U_{1}\right) \cap N_{q_{1}} .
$$

If $\operatorname{dim}_{\mathbf{R}} L_{q_{0}}^{\prime \prime}=0$ for some $q_{0} \in U \cap W$, then since $U_{1}$ is integral at $q_{0}, T_{q_{0}}^{\mathbb{C}}\left(U_{1}\right)$ $=\{0\}$ and $\operatorname{dim}_{\mathbf{R}} U_{1}=2$, this is impossible, so $\operatorname{dim}_{\mathbf{R}} L_{q}^{\prime \prime}=1$ for all $q \in$ $U_{1}$. Again, we argue as before, and we obtain a contradiction. Thus $U_{1}$ is nowhere totally real. Hence $\operatorname{dim}_{\mathbb{C}} T_{q}^{\mathbb{C}}\left(U_{1}\right)=1$ for all $q \in U_{1}$. Since $U_{1}$ is integral, this again gives a contradiction, by [1] and the fact that $U_{1}$ is of holomorphic dimension zero.

Now let us show the second part of Lemma 9.

Let $F_{1}=\left(f_{1}, \ldots, f_{n}\right)$ be a non-singular real-analytic parametrization of $Z$ defined near 0 in $\mathbf{R}^{s}(s=2 n-4)$ so that $F_{1}^{\prime}(0)=p$. Let

$$
\Phi_{j}(u)=\sum_{k=1}^{n} \frac{\partial r}{\partial z_{k}}\left(F_{1}(u)\right) \cdot \frac{\partial f_{k}}{\partial u_{j}}, \quad(1 \leq j \leq s)
$$

$r$ here is a defining function for $D$. Note that since $\left\{\Phi_{j}\right\}_{j=1}^{s}$ are real-analytic functions then

$$
Z^{\prime \prime}=\left\{F_{1}(u) \in Z \cap W: \Phi_{j}(u)=0 \text { for all }(1 \leq j \leq s)\right\}
$$


is a real-analytic subset of $Z$. This finishes the proof of Lemma 9.

Because the union of two real-analytic sets is a real-analytic set, we conclude that $Z^{\prime} \cup Z^{\prime \prime}$ is a real-analytic subset of $Z$. Therefore by Theorem 6 , and shrinking $U$ if necessary, we may write: $\left(Z^{\prime} \cup Z^{\prime \prime}\right) \cap U=\Omega_{2 n-5} \cup \cdots \Omega_{0}$, where each $\Omega_{t},(0 \leq t \leq 2 n-5)$, is a finite disjoint union of $t$-dimensional real-analytic submanifolds of $\partial D \cap U$, and of holomorphic dimension zero.

Let $Z^{\prime \prime \prime}=(Z \cap W) \backslash\left(Z^{\prime} \cup Z^{\prime \prime}\right)$.

Arguing as above, we conclude that $Z^{\prime \prime \prime}$ is a submanifold of $Z$ with $T_{q}\left(Z^{\prime \prime \prime}\right) \cap N_{q}=\{0\}$, for all $q$ near $p$.

Continuing this way, each time we stratify the sets:

$$
\left\{q \in \Theta \cap W: \operatorname{dim}_{\mathbf{R}} L_{q}^{(j)}=1\right\},
$$

and

$$
\{q \in \Theta \cap W: \Theta \text { is integral at } q\}
$$

where $\Theta \subset w(\partial D)$ is any submanifold of $\partial D \cap U$ of holomorphic dimension zero, and $\operatorname{dim}_{\mathbf{R}} \Theta \leq 2 n-5$, until its tangent space contains no weakly pseudoconvex tangent directions.

Finally, we may write $w(\partial D) \cap U$, for $U$ sufficiently small neighborhood of $p$ as :

$$
w(\partial D) \cap U=\Upsilon_{2 n-3} \cup \cdots \Upsilon_{0},
$$

where each $\Upsilon_{i},(0 \leq i \leq 2 n-3)$ is a finite disjoint union of real-analytic submanifolds of $\partial D \cap U$ with

$$
T_{q}\left(\Upsilon_{i}\right) \cap N_{q}=\{0\}
$$

for all $q \in \Upsilon_{i}$.

All it remains to show in part (a) is that the $\Upsilon_{i},(0 \leq i \leq 2 n-4)$ can be modified to obtain $C R$ manifolds. We note that we have already shown above that the $(2 n-3)$-dimensional manifolds are $C R$. We will show this and part (b) simultaneously using the following procedure.

Let $\Upsilon$ be a component of $\Upsilon_{2 n-4}$.

We put $\eta=\max \left\{\operatorname{dim}_{\mathbb{C}} T_{q}^{\mathbb{C}}(\Upsilon): q \in \Upsilon\right\}$, and let $E=\{q \in \Upsilon$ : $\left.\operatorname{dim}_{\mathbb{C}} T_{q}^{\mathbb{C}}(\Upsilon)=\eta\right\}$

We will show below that $E$ is a real-analytic subset of $\Upsilon$. Suppose for a moment this is true. We consider two cases:

If Int $(E)_{\Upsilon} \neq \emptyset$, then since $\Upsilon$ is connected, $E=\Upsilon$, and hence $\Upsilon$ is a $C R$ manifold.

If $\operatorname{Int}(E)_{\Upsilon}=\emptyset$, then by stratifying $E$ using Theorem 6 , we get lower dimensional manifolds. The complement $E_{1}$ of $E$ in $\Upsilon$ is an open subset of $\Upsilon$ with $\operatorname{dim}_{\mathbb{C}} T_{q}^{\mathbb{C}}\left(E_{1}\right) \leq \eta-1$, for all $q \in E_{1}$. We repeat the procedure above, replacing $\Upsilon$ by $E_{1}$. We continue this way, stratifying each time the 
sets of maximal complex dimension until we obtain an open subset $\Sigma$ of $\Upsilon$ with constant $C R$ dimension.

Now let us show that $E$ is a real-analytic subset of $\Upsilon$.

Suppose that $\Upsilon$ is given locally by $\Upsilon=\left\{q: r_{i}(q)=0,(1 \leq i \leq 4)\right\}$, where $r_{i}$ are real-analytic functions on $\Upsilon$. We know that

$$
\operatorname{dim}_{\mathbb{C}} T_{q}^{\mathbb{C}}(\Upsilon)=n-\operatorname{Rank}_{\mathbb{C}}\left[\frac{\partial r_{i}}{\partial z_{j}}(p)\right]_{\substack{1 \leq i \leq 4 \\ 1 \leq j \leq n}} .
$$

Now, if $q \in \Upsilon$, then

$$
\begin{aligned}
q \in E & \Longleftrightarrow \operatorname{dim}_{\mathbb{C}} T_{q}^{\mathbb{C}}(\Upsilon)=\eta>\eta-1 \\
& \Longleftrightarrow \operatorname{Rank}_{\mathbb{C}}\left[\frac{\partial r_{i}}{\partial z_{j}}(q)\right]<n-(\eta-1) \\
& \Longleftrightarrow \operatorname{det}\left(B_{q}\right)=0, \text { for every }(n-\eta+1) \times(n-\eta+1) \text { submatrix } B_{q} \\
& \text { of }\left[\frac{\partial r_{i}}{\partial z_{j}}(q)\right]_{\substack{1 \leq i \leq 4 \\
1 \leq j \leq n}} .
\end{aligned}
$$

Thus $E$ is a real-analytic subset of $\Upsilon$. This completes the proof of part (a).

Let $\Sigma$ be the set described above, and let $G=\{q \in \Sigma: \Sigma$ is integral at $q\}$. By the same argument as in Lemma $9, G$ is a real-analytic subset of $\Sigma$. As above there are two cases to consider:

If Int $(G)_{\Sigma} \neq \emptyset$, then $G=\Sigma$, and so $\Sigma$ is integral.

If Int $(G)_{\Sigma}=\emptyset$, then we apply Theorem 6 to stratify $G$, and we obtain lower dimensional manifolds. Then we consider the set $G^{\prime}=\Sigma \backslash G$, which is an open subset of $\Sigma$ with $G^{\prime}$ not integral at $q$ for all $q \in G^{\prime}$. Furthermore, we note that for all $q \in G^{\prime}, \operatorname{dim}_{\mathbb{C}} T_{q}^{\mathbb{C}}\left(G^{\prime}\right)=\operatorname{dim}_{\mathbb{C}} T_{q}^{\mathbb{C}}(\Sigma)$ and hence $G^{\prime}$ is a $C R$ manifold. This finishes the argument for the $(2 n-4)$-dimensional submanifolds of $\partial D \cap U$.

We carry out the same reasoning for the real-analytic submanifolds $\Delta$ of $\partial D \cap U$ of dimension less than or equal to $(2 n-5)$, as we did for $\Upsilon$, stratifying first the set of points in $\Delta$ where the complex tangent space of $\Delta$ has maximal dimension until we obtain an open subset $\Delta^{\prime}$ of $\Delta$ which is a $C R$ manifold, and then stratifying the set of points in $\Delta^{\prime}$ where $\Delta^{\prime}$ is integral. We do this inductively until all the strata of $w(\partial D)$ satisfy the properties (a) and (b) stated in the theorem.

Part (c) follows immediately from the techniques used in Theorem 6. This concludes the proof of Theorem 5 .

Remark. Fornaess and Overlid gave in [8] a global decomposition of $w(\partial D)$ for pseudoconvex domains with real-analytic boundaries in $\mathbb{C}^{2}$. In fact, they have shown that $w(\partial D)$ for such domains can be written as:

$$
w(\partial D)=S_{0} \cup S_{1} \cup S_{2}
$$


where each $S_{j}$ is a finite disjoint union of $j$-dimensional totally real realanalytic submanifolds of $\partial D$. For convex domains with real-analytic boundaries in $\mathbb{C}^{2}$, Theorem 5 tells us that the maximal strata of $w(\partial D)$ is a curve, i.e. $S_{2}=\emptyset$. That $S_{2}$ is empty in $\mathbb{C}^{2}$ is of course an immediate consequence of linear regularity.

\section{Peak sets.}

Example 10. Let $D=\left\{z=\left(z_{1}, z_{2}, z_{3}\right) \in \mathbb{C}^{3}: r(z)=\operatorname{Re} z_{3}+\left|z_{1}\right|^{2}+\left|z_{2}\right|^{4}\right.$ $<0\}$. It is obvious that $D$ is a convex domain with real-analytic boundary, and

$$
w(\partial D)=\left\{z \in \partial D: z_{2}=0, \operatorname{Re} z_{3}=-\left|z_{1}\right|^{2}\right\}
$$

Let

$$
\begin{aligned}
K=\left\{z \in \partial D: \operatorname{Re} z_{2}=\operatorname{Im} z_{1}=\operatorname{Im} z_{3}=0\right\} \cup \\
\left\{z \in \partial D: \operatorname{Im} z_{1}=\operatorname{Im} z_{2}=\operatorname{Im} z_{3}=0\right\} .
\end{aligned}
$$

It is easy to see that $K$ is a peak set for $A^{\infty}(D)$ with strong support function $f(z)=z_{3}+z_{1}^{2}+z_{2}^{4}$. We note that $K \cap w(\partial D)=\left\{z \in \partial D: z_{2}=\operatorname{Im} z_{3}=\operatorname{Im} z_{1}\right.$ $\left.=0, \operatorname{Re} z_{3}=-\left(\operatorname{Re} z_{1}\right)^{2}\right\}$ is an integral curve which points in the strongly pseudoconvex direction. We patch peak functions near this curve.

We include a brief sketch of the proof of Theorem 11, which was proved by the author in [2], it shows that the intersection of a peak set and any strata of $w(\partial D)$ described in Theorem 5 is locally contained in integral manifolds. In our situation, we will patch peak functions along these integral curves.

Theorem 11. Suppose $D \subset \subset \mathbb{C}^{n}$ is a convex domain with real-analytic boundary. Assume $K$ is a compact subset of $\partial D$ which is locally a peak set for $A^{\infty}(D)$. Let $S$ be any strata of $w(\partial D)$ as in Theorem 5, and suppose $\operatorname{dim}_{\mathbf{R}} S=2 t+\lambda$, where $\operatorname{dim}_{\mathbf{C}} T_{q_{0}}^{\mathbb{C}}(S)=t$ for all $q_{0} \in S$. Suppose $p \in K \cap S$. Then there exist a neighborhood $U \subset \mathbb{C}^{n}$ of $p$, a holomorphic change of coordinates in $U$ in which $p=0$ and $S \subset \mathbb{C}^{t+\lambda} \times\{0\}$, a neighborhood $U^{\prime} \subset \mathbb{C}^{t+\lambda}$ of 0 , a strongly pseudoconvex domain $\Omega \subset \subset U^{\prime}$, a locally peak set $\widetilde{L} \subset \partial \Omega \cap V^{\prime}$, where $V^{\prime} \subset \subset U^{\prime}$ is a neighborhood of zero, and a totally real smooth $\widetilde{M} \subset \partial \Omega \cap V^{\prime}$ so that:

(a) $K \cap S \cap V \subseteq \widehat{L} \subset \widehat{M} \subset(\partial \Omega \times\{0\}) \cap V^{\prime \prime} \subset \partial D \cap V$, where $\widehat{L}=$ $\widetilde{L} \times\{0\}, \widehat{M}=\widetilde{M} \times\{0\}, V^{\prime \prime}=V^{\prime} \times\{0\}$, and $V \subset \subset U$ is a neighborhood of 0 .

(b) $T_{q}(\widehat{M}) \subseteq T_{q}^{\mathbb{C}}(\partial D)$ for all $q \in \widehat{M}$.

(c) $\operatorname{dim}_{\mathbf{R}} \widehat{M} \leq n-2$. 
Sketch of Proof. Assume $T_{p}(S) \nsubseteq T_{p}^{C}(\partial D)$. Since $S$ is a real-analytic CR manifold, then by Rossi's theorem [17], there exists a neighborhood $U$ of $p$ in $C^{n}$ and a biholomorphic map $\Phi: U \longrightarrow \mathbb{C}^{n}$ so that $\Phi(p)=0$ and $\Phi(U \cap S) \subset \mathbb{C}^{t+\lambda} \times\{0\}$. Note that Rossi's theorem enables us to put real-analytic $\mathrm{CR}$ submanifolds of $\mathbb{C}^{n}$ into lower dimensional $\mathbf{C}^{\mathbf{k}}(k<n)$. Let $z \in U$, and $\Phi(z)=\left(z^{\prime}, z^{\prime \prime}\right)$, with $z^{\prime}=\left(z_{1}, \ldots, z_{t+\lambda}\right)$, denotes the new holomorphic change of coordinates near 0 , where $z_{t+\lambda}=u+i v$ is the complex normal to $\partial D$. We assume that the new manifold obtained under $\Phi$ that sits in $\mathbf{C}^{\mathbf{t}+\lambda} \times\{0\}$ is also denoted by $S$. We define the function $\rho$ by:

$$
\rho\left(z^{\prime}\right)=r \circ h\left(z^{\prime}\right),
$$

where $h\left(z^{\prime}\right)=\left(z^{\prime}, 0, \ldots, 0\right)$. Let $U^{\prime}$ be a neighborhood of $0^{\prime}$ in $C^{t+r}$, and put

$$
\Omega=\left\{z^{\prime} \in U^{\prime}: \rho\left(z^{\prime}\right)<0\right\} .
$$

Note that $\Omega$ is a bounded domain in $U^{\prime}$, and $S$ is locally contained in $(\partial \Omega \times$ $\{0\}) \cap U^{\prime}$, where

$$
\partial \Omega \cap U^{\prime}=\left\{z^{\prime} \in U^{\prime}: \rho\left(z^{\prime}\right)=0\right\} .
$$

It is shown in [2] that $\rho$ is a defining function for $\Omega$,and that $\Omega$ is strongly pseudoconvex at $0^{\prime}$. Finally, we show that there exists locally a peak set $\widetilde{L}$ for $A^{\infty}(\Omega)$ so that

$$
K \cap S \cap V \subseteq \widetilde{L} \times\{0\},
$$

where $V \subset U$ is a neighborhood of 0 . Let $f$ be a strong support function for $K \cap \bar{V}$, where $V \subset U$ is an open neighborhood of 0 in $C^{n}$. Define the function $g$ by

$$
g\left(z^{\prime}\right)=f \circ h\left(z^{\prime}\right)
$$

Put

$$
\widetilde{L}=\left\{z ; \in \bar{\Omega} \cap V^{\prime}: g\left(z^{\prime}\right)=0\right\},
$$

with $V^{\prime} \subset U^{\prime}$ as a neighborhood of $0^{\prime}$. We claim that $g$ is a strong support function for $\widetilde{L} \cap \bar{V}^{\prime}$. It is obvious that $g \in A^{\infty}(\Omega) . g \not \equiv 0$ on $\bar{\Omega} \cap V^{\prime}$. To see this, assume to the contrary that $g \equiv 0$ on $\bar{\Omega} \cap V^{\prime}$. Then by the Chain Rule:

$$
\frac{\partial f}{\partial u}(0)=0 \text {. }
$$

But this is absurd because $\operatorname{Re} f$ is pluriharmonic in $D$, nonconstant, and has a local minimum at 0 , so by Hopf lemma,

$$
\frac{\partial(\operatorname{Re} f)}{\partial u}(0)<0 .
$$

We note that if $\operatorname{Re} g=0$, then $g=0$, and therefore $\operatorname{Re} g<0$ on $\bar{\Omega} \backslash \widetilde{L} \cap \bar{V}^{\prime}$. In addition, $\widetilde{L} \subset \partial \Omega \cap V^{\prime}$ which follows from the maximum modulus principle. Let $z \in K \cap S \cap V$, with $z=\left(z_{1}, \ldots, z_{t+r}, 0, \ldots, 0\right)=\left(z^{\prime}, 0, \ldots, 0\right)$. Then, $g\left(z^{\prime}\right)=f \circ h\left(z^{\prime}\right)=f\left(z^{\prime}, 0, \ldots, 0\right)=0$ since $z \in K$. So $z^{\prime} \in \widetilde{L}$, and $\left(z^{\prime}, 0\right) \in$ 
$\widetilde{L} \times\{0\}$. Thus $K \cap V \subset \widetilde{L} \times\{0\}$. Now, we verify properties (a)-(c) of the theorem. Since $\Omega$ is a strongly pseudoconvex domain near $0^{\prime}$, and $\widetilde{L}$ is locally a peak set for $A^{\infty}(\Omega)$, then by Theorem $1((1) \Rightarrow(3))$ due to Chaumat and Chollet, we obtain a totally real-submanifold $\widetilde{M}$ of $\partial \Omega \cap V^{\prime}$, if $V^{\prime}$ is small enough so that:

$$
K \cap S \cap V \subseteq \widetilde{L} \times\{0\} \subset(\partial \Omega \times\{0\}) \cap V^{\prime \prime} \subset \partial D \cap V,
$$

where $V^{\prime \prime}=V^{\prime} \times\{0\}$, and for sufficiently small neighborhood $V \subset U$ of 0 . Furthermore, since $\widetilde{L}$ is locally a peak set for $A^{\infty}(\Omega)$, then for all $q \in \widehat{M}$,

$$
T_{q}(\widehat{M}) \subseteq T_{q}^{C}(\partial D)
$$

where $\widehat{M}=\widetilde{M} \times\{0\}$. Also,

$$
\operatorname{dim}_{\mathrm{R}} \widehat{M}=t+r-1 \leq(n-1)-1=n-2 .
$$

Finally, if $T_{p}(S) \subseteq T_{p}^{C}(\partial D)$, then $S$ must be an integral manifold by part (b) of Theorem 5. Also, since $S$ is a CR-submanifold of $\partial D, \mathrm{~S}$ must be totally real, and therefore the preceding proof is easily modified. This completes the sketch of the proof.

We recall from $[\mathbf{2}]$ the following:

Theorem 12. Suppose $D \subset \subset \mathbb{C}^{n}$ is a convex domain with real-analytic boundary. Assume $K$ is a peak set for $A^{\infty}(D)$, and $L$ is a compact subset of $K$. Then $L$ is a peak set for $A^{\infty}(D)$.

Proposition 13 (Almost-holomorphic extension; [11]). Suppose $\widehat{M} \subset \partial D$ $\cap U$ is a totally real submanifold, where $U$ is an open subset of $\mathbb{C}^{n}$. Let $\chi$ be a $C^{\infty}$ function in $\widehat{M}$. Then there exists a $C^{\infty}$ function $\tilde{\chi}$ in $U$ so that:

(1) $\tilde{\chi}=\chi$ on $\widehat{M}$.

(2) $\bar{\partial} \widetilde{\chi}$ vanishes to infinite order along $\widehat{M}$, i.e. $D^{\alpha}(\bar{\partial} \widetilde{\chi}) \equiv 0$ along $\widehat{M} \cap U$ for each multi-index $\alpha$.

(3) $\tilde{\chi}$ is locally constant near where $\chi$ is locally constant.

(4) First derivatives of $\tilde{\chi}$ vanishes on $\widehat{M}$ in directions perpendicular to $T(\widehat{M})+J T(\widehat{M})$.

The next proposition goes back to Chaumat and Chollet [4]. Proposition 14 allows us to construct peak functions from the functions stated there.

Proposition 14. Suppose $D \subset \mathbb{C}^{n}$ is a bounded pseudoconvex domain with smooth boundary. Let $E$ be a compact subset of $\partial D, W$ a neighborhood of $E$ in $\mathbb{C}^{n}$, and $\rho$ a non-negative continuous function on $W$ which vanishes on E. Suppose that there exists a function $G \in C^{\infty}(W \cap \bar{D})$ such that:

(a) $E=\{z \in W \cap \bar{D}: G(z)=0\}$. 
(b) For each $\alpha \in \mathbf{N}^{n}$, and for each $k \in \mathbf{N}$, there exists a constant $C_{\alpha k}>0$ such that for each $z \in W \cap \bar{D}$

$$
\left|D^{\alpha}(\bar{\partial} G(z))\right| \leq C_{\alpha k}[\rho(z)]^{k} .
$$

(c) There exists a constant $c>0$ so that for all $z \in \bar{D} \cap W$,

$$
\operatorname{Re} G(z) \geq c \rho(z) .
$$

Then $E$ is a peak set for $A^{\infty}(D)$.

Notation. For $R>0$, let $S_{R}(f)=f-R f^{2}$, where $f$ is a strong support function for a closed subset $K \subset \partial D$. We note that

$$
\operatorname{Re}\left(S_{R}(f)\right)=(\operatorname{Re} f)(1-R(\operatorname{Re} f))+R(\operatorname{Im} f)^{2} .
$$

Moreover, for a small neighborhood $U$ of $K$,

$$
S_{R}(f)=0 \text { on } K \text { and } \operatorname{Re}\left(S_{R}(f)\right)>0 \text { on }(\bar{D} \cap U) \backslash K \text {. }
$$

\section{Proof of the main result.}

We first state and prove the Patching Lemma, this will be the main tool in proving the main result.

Patching Lemma 15. Suppose $D \subset \subset \mathbb{C}^{3}$ is a convex domain with realanalytic boundary. Let $f_{i}(i=1,2)$ be strong support functions for $K \cap \bar{U}_{i}$, where $U_{i}$ are open subsets of $\mathbb{C}^{3}$ with $U_{1} \cap U_{2} \neq \emptyset$. Put $K_{i}=K \cap \bar{U}_{i}$, $(i=1,2)$. Let $p \in K_{1} \cap K_{2} \cap w(\partial D)$. Let $U \subset \mathbb{C}^{3}, U^{\prime} \subset \mathbb{C}^{2}, \Omega, \widehat{L}$ and $\widehat{M}$ be as in Theorem 11. Assume $K_{1} \cap K_{2} \cap w(\partial D) \cap U=\widehat{M}$, and $\operatorname{dim}_{\mathbf{R}} \widehat{M}=1$. Let $R$ be a sufficiently large positive number. Then there exist a neighborhood $V \subset \subset U$ of $p$ with $\partial V \cap \widehat{M}=\left\{p_{1}, p_{2}\right\}$, a smooth manifold $M \subset U$ containing $\widehat{M}$, and a function $g \in C^{\infty}(\bar{D} \cap V)$ with the following properties:

(a) $g \equiv 0$ on $K_{1} \cap K_{2} \cap V$.

(b) $\operatorname{Re} g>0$ on $(\bar{D} \cap V) \backslash\left(K_{1} \cap K_{2} \cap V\right)$.

(c) $\operatorname{Re} g\left(q^{\prime}\right) \geq c d^{2}\left(q^{\prime}, M\right)$ when $q^{\prime} \in \bar{D} \cap V$ and where $c$ is a positive constant.

(d) $D^{\alpha}\left(\bar{\partial} g\left(q^{\prime}\right)\right)=0$ for each $q^{\prime} \in \bar{D} \cap V \cap M$ and for each multi-index $\alpha$.

(e) $g=S_{R}\left(f_{i}\right)$ near $p_{i}(i=1,2)$.

Proof. Choose a holomorphic coordinate system $\left(z_{1}, z_{2}, z_{3}\right)$ in $U$ so that $p=0$ as described in Theorem 11. Let $\widetilde{M}$ be as in Theorem 11, and assume without loss of generality that $\widetilde{M} \subset \mathbb{C}^{2}$.

Let $\gamma:(-3,3) \longrightarrow \partial \Omega$ be a non-singular parameterization of $\widetilde{M}$ so that $\gamma(0)=(0,0)$. Choose a $C^{\infty}$-cut off function $\chi: \widetilde{M} \longrightarrow[0,1]$ so that:

$$
\chi(\gamma(t))=1 \text { for } t \geq 1
$$


and

$$
\chi(\gamma(t))=0 \text { for } t \leq-1
$$

Proposition 13 guarantees the existence of an almost-holomorphic extension $\chi^{\prime}$ of $\chi$ defined in $U^{\prime}$ so that properties $(1) \rightarrow(4)$ hold. We extend $\chi^{\prime}$ trivially to get a function $\tilde{\chi}$ defined on $U^{\prime} \times \mathbb{C}$, and $\tilde{\chi}\left(z_{1}, z_{2}, z_{3}\right)=\chi^{\prime}\left(z_{1}, z_{2}\right)$.

We put

$$
g=\tilde{\chi} S_{R}\left(f_{2}\right)+(1-\tilde{\chi}) S_{R}\left(f_{1}\right) .
$$

Let $V \subset \subset U$ be a small neighborhood of $L^{\prime}=\{(\gamma(t), 0):-2<t<2\}$ so that $\partial V \cap \widehat{M}=\left\{p_{1}, p_{2}\right\}$, with $p_{1}=(\gamma(-2), 0)$ and $p_{2}=(\gamma(2), 0)$. It is clear that $g \in C^{\infty}(\bar{D} \cap V)$.

Let

$$
M=\widetilde{M} \times \mathbb{C} .
$$

Then $M$ is a smooth submanifold of $\mathbb{C}^{3}$ containing $\widetilde{M}$.

Now let us show properties $(\mathrm{a}) \rightarrow(\mathrm{e})$.

(a) Since $S_{R}\left(f_{i}\right)=0(i=1,2)$ on $K_{1} \cap K_{2} \cap V$, part (a) follows.

(e) Is obvious because of part (3) of Proposition 13.

(d) Since $\bar{\partial} \chi^{\prime}$ vanishes to infinite order along $\widetilde{M}$, then $\bar{\partial} \widetilde{\chi}$ vanishes to infinite order along $M$ as well, this gives part (d).

(b) To show part (b), we assume for a moment that (c) holds. Then if $V$ is small enough, $\operatorname{Re} g>0$ on $(\bar{D} \cap V) \backslash(M \cap V)$, in addition, on $M \cap V$ we have

$$
\operatorname{Re} g=\chi S_{R}\left(f_{2}\right)+(1-\chi) S_{R}\left(f_{1}\right),
$$

and so $\operatorname{Re}\left(S_{R}\left(f_{i}\right)\right)>0$ on $(\bar{D} \cap V \cap M) \backslash\left(K_{1} \cap K_{2} \cap V\right)$. Therefore (b) follows.

All it remains to show is part (c).

Pick $q \in \widehat{M}=\widetilde{M} \times\{0\}$. Since $\widehat{M}$ is totally real, we can make a holomorphic change of coordinates in $\mathbb{C}^{3}$ near $q$, that we still denote by $\left(z_{1}, z_{2}, z_{3}\right)$ so that $q=0$ and $T_{0}(\widetilde{M})=\left\{\left(z_{1}, z_{2}\right): y_{1}=z_{2}=0\right\}$. Assume also that $T_{0}(\partial \Omega)=\left\{\left(z_{1}, z_{2}\right): u=0\right\}$, and $T_{0}(\partial D)=\left\{\left(z_{1}, z_{2}, z_{3}\right): u=0\right\}$. Here $z_{j}=$ $x_{j}+i y_{j},(j=1,3)$ and $z_{2}=u+i v$.

Fix $i$, and let $h\left(z_{1}, z_{2}\right)=f_{i}\left(z_{1}, z_{2}, 0\right)$. By the proof of Theorem 11, we know that $h \in A^{\infty}(\Omega)$ is a strong support function for $\widetilde{L} \cap \bar{U}^{\prime}$ with $U^{\prime}$ a small neighborhood of $(0,0)$. Since $\Omega$ is a strongly pseudoconvex domain, and $K_{1} \cap K_{2} \cap w(\partial D) \cap U=\widehat{M}=\widetilde{M} \times\{0\}$ is an integral curve of $\partial \Omega \times\{0\}$ by assumption, Proposition 9 in [5] guarantees that

$$
B=\frac{\partial^{2} h}{\partial y_{1}^{2}}(0,0)=\frac{\partial^{2} f_{i}}{\partial y_{1}^{2}}(0,0,0)>0 .
$$


Furthermore, we deduce from the Cauchy-Riemann equations and the Hopf lemma that,

$$
\frac{\partial\left(\operatorname{Re} f_{i}\right)}{\partial u}(q)=\frac{\partial\left(\operatorname{Im} f_{i}\right)}{\partial v}(q)=\epsilon<0 .
$$

Using (11) we get by Taylor expanding $\operatorname{Re}\left(S_{R}\left(f_{i}\right)\right)$ at $q$ in directions perpendicular to $M$ for $q^{\prime} \in \bar{D}$ near $q$ :

$$
\operatorname{Re}\left(S_{R}\left(f_{i}\right)\right)\left(q^{\prime}\right)=\epsilon u-R \epsilon^{2} u^{2}+R \epsilon^{2} v^{2}+Q\left(z_{1}, z_{2}\right),
$$

where $Q\left(z_{1}, z_{2}\right)$ is of second order and is independent of $R$. This shows that we can control $\operatorname{Re} S_{R}\left(f_{i}\right)$ in $J \vec{n}$, and $\vec{n}$ is the outer normal vector to $\partial D$.

By (9) we have,

$$
\begin{aligned}
\operatorname{Re} g= & (\operatorname{Re} \tilde{\chi})\left(\operatorname{Re} S_{R}\left(f_{2}\right)+\operatorname{Re}(1-\tilde{\chi}) \operatorname{Re} S_{R}\left(f_{1}\right)\right) \\
& +\operatorname{Im} \tilde{\chi}\left(\operatorname{Im} S_{R}\left(f_{1}\right)-\operatorname{Im} S_{R}\left(f_{2}\right)\right) .
\end{aligned}
$$

Let $G_{1}$ be the sum of the first two terms and $G_{2}$ the last term.

Using (10), (12) and since $\left.\tilde{\chi}\right|_{M}=\chi$, then the Taylor expansion of $G_{1}$ in directions perpendicular to $M$ is:

$$
\operatorname{Re} G_{1}\left(q^{\prime}\right) \geq A u^{2}+B y_{1}^{2}+C_{R} v^{2}
$$

for all $q^{\prime} \in \bar{D}$ near $q$, and for some positive constants $A$, and $C_{R}$.

By part (4) of Proposition 13 and the Taylor expansion of $G_{2}$ at $q^{\prime} \in \bar{D}$ near $q$ in directions perpendicular to $M$, we get

$$
\operatorname{Re} G_{2}=E y_{1} v+\text { error terms, }
$$

where $E$ is independent of $R$.

We observe that for $R$ large enough, $A u^{2}+B y_{1}^{2}+C_{R} v^{2} \gg E y_{1} v$. Thus for all $q^{\prime} \in \bar{D}$ near $q$, it follows that,

$$
\operatorname{Re} g\left(q^{\prime}\right) \geq A u^{2}+B y_{1}^{2}+C_{R} v^{2} .
$$

Since $L^{\prime}$ is compact, we may conclude that for all $q^{\prime} \in \bar{D} \cap V$, and some positive constant $c$,

$$
\operatorname{Re} g\left(q^{\prime}\right) \geq c d^{2}\left(q^{\prime}, M\right) .
$$

This finishes the proof of the Patching Lemma.

Theorem 16. Suppose $D \subset \subset \mathbb{C}^{3}$ is a convex domain with real-analytic boundary. Then a compact subset $K$ of $\partial D$ is locally a peak set for $A^{\infty}(D)$ if and only if $K$ is a peak set for $A^{\infty}(D)$.

Proof. Choose open sets $\left\{U_{i}\right\}_{i=1}^{m}$ in $\mathbb{C}^{3}$ so that $K \cap w(\partial D) \subset \cup_{i=1}^{m} U_{i}$, and $K \cap \bar{U}_{i}$ is a peak set for $A^{\infty}(D)$ with strong support function $f_{i},(1 \leq i \leq m)$. 
We apply Theorem 11 and the decomposition of $w(\partial D)$ given by Theorem 5 to our situation, and since $K \cap w(\partial D)$ is compact, we deduce that

$$
K \cap w(\partial D) \subseteq\left(\cup_{j=1}^{l} \widehat{M}\right) \cup M_{0},
$$

where $\widehat{M}_{j}(1 \leq j \leq l)$ are disjoint integral curves, and $M_{0}$ is a finite set.

We may assume that $M_{0} \cap \partial U_{i}=\emptyset$ for all $i$, otherwise we may shrink $U_{i}$ to obtain a smaller neighborhood $W_{i}$ so that $K \cap \bar{W}_{i}$ is a peak set for $A^{\infty}(D)$, this is because compact subsets of peak sets for $A^{\infty}(D)$ are peak sets for $A^{\infty}(D)$ by Theorem 12 .

Fix $i$ and $j$. Let $p \in K \cap w(\partial D) \cap \widehat{M}_{j} \cap \partial U_{i}$, and choose $k$ so that $p \in U_{k}$. We will consider two cases.

Case 1. $K \cap w(\partial D)$ is a connected subset of $\widehat{M}_{j}$ near $p$.

Case 2. $K \cap w(\partial D)$ is not a connected subset of $\widehat{M}_{j}$ near $p$.

Suppose Case 1 occurs. Apply the Patching Lemma 15, with strong support function $f_{i}$ and $f_{k}$ for $K \cap \bar{U}_{i}$ and $K \cap \bar{U}_{k}$ respectively to obtain a $C^{\infty}$-function $g_{i j}$ defined in a small neighborhood $V_{i j}$ of $p$ so that $\partial V_{i j} \cap \widehat{M}_{j}=\left\{p_{i j}, p_{k j}\right\}$, and

$$
g_{i j}= \begin{cases}S_{R}\left(f_{i}\right) & \text { near } p_{i j} \\ S_{R}\left(f_{k}\right) & \text { near } p_{k j} .\end{cases}
$$

We do this for each $i$ and $j$.

Suppose Case 2 occurs. Choose disjoint neighborhoods $\left\{W_{s}\right\}_{s=1}^{2}$ of $K \cap$ $w(\partial D)$ in $U_{i} \cup U_{k}$ so that $K \cap W_{s}(s=1,2)$ is a peak set for $A^{\infty}(D)$. Let $U$ be a neighborhood of $K \backslash\left(W_{1} \cup W_{2}\right)$ such that $U \cap w(\partial D)=\emptyset$ in $W_{s}$ $(s=1,2)$. In [7] Fornaess and Henriksen showed that if a compact subset $K_{1}$ is contained in the boundary of a strongly pseudoconvex domain in $\mathbb{C}^{3}$ and $K_{1}$ is locally contained in integral manifolds, then there exists an integral manifold containing all of $K_{1}$.

Let $K_{1}=(K \cap U) \backslash\left(W_{1} \cup W_{2}\right)$. Since $K_{1}$ consists only of strongly pseudoconvex boundary points, then by the fact mentioned above, we obtain that $K_{1}$ is globally contained in a finite disjoint union of integral manifolds.

Thus, if $K \cap\left(U \cap\left(W_{1} \cup W_{2}\right)\right) \neq \emptyset$, then we may use the techniques in [7] to patch peak functions.

We are now prepared to construct a function $G$ and a function $\rho$ satisfying conditions (a) $\rightarrow$ (c) of Proposition 14 .

Put $E=K$.

Let

$$
G= \begin{cases}g_{i j} & \text { in } V_{i j} \\ S_{R}\left(f_{i}\right) & \text { near } p_{i j} \\ S_{R}\left(f_{k}\right) & \text { near } p_{k j} .\end{cases}
$$


Pick a neighborhood $W$ of $E$ so that if $z \in \bar{D} \cap W$, then

$$
G(z)=0 \text { if and only if } z \in E \text {. }
$$

Let $M_{j}$ be the smooth manifold in $\mathbb{C}^{3}$ obtained by the Patching Lemma 15 so that $M_{j} \supset \widehat{M}_{j}$, and for all $q \in \bar{D} \cap W$ near $p$,

$$
\operatorname{Re} G(q) \geq c d^{2}\left(q, M_{j}\right) .
$$

We put $\rho(z)=d^{2}\left(z, M_{j}\right)$ in a neighborhood of $\widehat{M}_{j}$ containing $p_{i j}$ and $p_{k j}$. Now let us verify the hypothesis of Proposition 14.

Part (a) follows from (15).

Part (b) is true for points between $p_{i j}$ and $p_{k j}$ by part (d) of the Patching Lemma 15 , and $G=S_{R}\left(f_{i}\right)$ or $G=S_{R}\left(f_{k}\right)$ away from such points, and hence $G$ is holomorphic in $D$. So, part (b) holds for all points in $\bar{D} \cap W$.

Part (c) follows since if $\rho(z)=d^{2}\left(z, M_{j}\right)$, and $G=g_{i j}$ in a neighborhood of $\widehat{M}_{j}$ that includes $p_{i j}$ and $p_{k j}$, then by (16)

$$
\operatorname{Re} G(z) \geq c d^{2}\left(z, M_{j}\right)
$$

for all $z \in \bar{D} \cap W$ near $p$. Away from such points we have, $\operatorname{Re} G=S_{R}\left(f_{l}\right) \geq$ $0(l=1,2)$, so we may choose $\rho=0$.

Thus, $K$ is a peak set for $A^{\infty}(D)$. This concludes the proof of the main result.

Remark. The main difficulty in proving Theorem 16 in the case $\mathbb{C}^{n}(n>3)$, is finding a generalization of the Patching Lemma 15 in higher dimensional convex domains $(n>3)$. For $n=3$, one only needed to patch peak functions along integral curves by Theorem 11, and these curves point in the strongly pseudoconvex directions. Further, the function $S_{R}(f)$ introduced above proved to be useful in this case. The author believes that the case $C^{n}$ $(n>3)$ is somewhat different, and would require the construction of a more complicated Patching Lemma.

\section{References}

[1] E. Bedborf and J.E. Fornaess, Complex manifolds in pseudoconvex boundaries, Duke Math. J., 48 (1981), 279-288.

[2] R. Belhachemi, Peak sets in real-analytic convex boundaries, Pacific J. Math., 185(2) (1998), 209-219.

[3] A. Bogess, CR manifolds and the tangential Cauchy-Riemann complex, Studies in Advanced Math., CRC Press, 1991.

[4] J. Chaumat and A. M. Chollet, Ensembles pics pour $A^{\infty}(D)$, Ann. Inst. Fourier, 29 (1979), 171-200.

[5] Caracterisation et proprietés des ensembles localement pics de $A^{\infty}(D)$, Duke Math. J., 47 (1980), 763-787. 
[6] K. Diederich and J.E. Fornaess, Pseudoconvex domains with real-analytic boundary, Ann. of Math., 107 (1978), 371-384.

[7] J.E. Fornaess, Plurisubharmonic defining functions, Pacific J. Math., 80 (1979), 381388.

[8] J.E. Fornaess and Ovrelid, Finitely generated ideals in $A(\Omega)$, Ann. Inst. Fourier (Grenoble), 33 (1983), 77-85.

[9] J.E. Fornaess and B. Henriksen, Characterization of global peak sets for $A^{\infty}(D)$, Math. Ann., 259 (1982), 125-130.

[10] M. Hakim and N. Sibony, Ensembles pics dans des domaines strictement pseudoconvex, Duke Math. J., 45 (1978), 601-617.

[11] F.R. Harvey and R.O. Wells, Holomorphic approximation and hyperfunction theory on a $C^{1}$ totally real submanifold of a complex manifold, Tran. Amer. Soc., 181 (1973), 273-292.

[12] A. Iordan, Peak sets in weakly pseudoconvex domains, Math. Z., 188 (1985), 171-188.

[13] S.G. Krantz and H.R. Parks, A primer of real-analytic functions, A series of Advanced Textbooks in Mathematics, Vol. 4, Birkhauser Verlag, 1992.

[14] A. Noell, Properties of peak sets in weakly pseudoconvex boundaries in $\mathbb{C}^{2}$, Math. Z., 186 (1984), 99-116.

[15] _ Local versus global convexity of pseudoconvex domains, Proceedings of symposia in Pure Mathematics, 52 (1991), Part 1.

[16] R.M. Range, Holomorphic functions and integral representations in several complex variables, Springer-Verlag, New York, 1986.

[17] H. Rossi, Differentiable submanifolds of complex Euclidean space, International Congress of Mathematics, Moscow, 1966.

[18] B.A. Taylor and D.L. Williams, The peak sets for $A^{m}$, Proc. Amer. Math. Soc., 24 (1970), 604-606.

Received November 12, 1996 and revised April 27, 1997.

Ryerson Polytechnic University

Department of Mathematics, Physics and Computer Science

Toronto Ontario M5B 2K3

CANADA

E-mail address: rbelhach@acs.ryerson.ca 